\title{
INTEGRALS FOR ASYMPTOTIC EXPANSIONS OF HYPERGEOMETRIC FUNCTIONS
}

\section{J. SLATER}

1. The integral for ordinary hypergeometric functions. In this paper I discuss integrals which provide explicit asymptotic expansions of generalized basic hypergeometric functions. The problem of asymptotic expansions for hypergeometric functions has been considered previously by C. S. Maijer [2], E. M. Wright [5], and, for basic functions, by G. N. Watson [4].

Let

$$
{ }_{A} F_{B}[(a) ;(b) ; z]=\sum_{n=0}^{\infty} \frac{\left(a_{1}\right)_{n}\left(a_{2}\right)_{n} \cdots\left(a_{A}\right)_{n} z^{n}}{\left(b_{1}\right)_{n}\left(b_{2}\right)_{n} \cdots\left(b_{B}\right)_{n} n !}
$$

where $(a)_{n}=a(a+1)(a+2) \cdots(a+n-1)$. Also let

$$
\Gamma[(a) ;(b)]=\frac{\Gamma\left(a_{1}\right) \Gamma\left(a_{2}\right) \cdots \Gamma\left(a_{A}\right)}{\Gamma\left(b_{1}\right) \Gamma\left(b_{2}\right) \cdots \Gamma\left(b_{B}\right)} .
$$

A dash will denote the omission of a vanishing factor in a sequence. Thus, $(a)^{\prime}-a_{r}$ denotes the sequence $a_{1}-a_{r}, a_{2}-a_{r}, \cdots, a_{r-1}-a_{r}$, $a_{r+1}-a_{r}, \cdots, a_{A}-a_{r}$.

It is known already (see [2] and [5]) that if

$$
\begin{aligned}
I(z)= & \frac{1}{2 \pi i} \int_{-i \infty}^{i \infty} \Gamma\left[\begin{array}{l}
(a)+s,(b)-s \\
(c)+s,(d)-s
\end{array}\right] z^{s} d s, \\
\sum_{A}(z)= & \sum_{\mu=1}^{A} z^{-a_{\mu} \Gamma}\left[\begin{array}{l}
(a)^{\prime}-a_{\mu},(b)+a_{\mu} \\
(c)-a_{\mu},(d)+a_{\mu}
\end{array}\right] \\
& \times_{B+C} F_{A+D-1}\left[\begin{array}{l}
(b)+a_{\mu}, 1+a_{\mu}-(c) ; \\
1+a_{\mu}-(a),(d)+a_{\mu} ;
\end{array}(-1)^{A+c_{z^{-1}}}\right], \\
\sum_{B}(z)= & \sum_{\nu=1}^{B} z^{b_{\nu}} \Gamma\left[\begin{array}{l}
(a)+b_{\nu},(b)^{\prime}-b_{\nu} \\
(c)+b_{\nu},(d)-b_{\nu}
\end{array}\right] \\
& \times_{A+D} F_{B+C-1}\left[\begin{array}{l}
\left.(a)+b_{\nu}, 1+b_{\nu}-(d) ;(-1)^{B+D_{z}}\right] \\
1+b_{\nu}-(b),(c)+b_{\nu} ;
\end{array}\right.
\end{aligned}
$$

then, provided that $\pi|A+B-C-D| / 2>|\arg z|$,

(i) $I(z)=\sum_{A}(z) \sim \sum_{B}(z)$ when $B+C<A+D$,

$$
\text { (ii) } I(z)=\sum_{B}(z) \sim \sum_{A}(z) \text { when } B+C>A+D \text {, }
$$

Received by the editors March 15, 1954 and, in revised form, June 14, 1954. 
and (iii) $I(1)=\sum_{A}(1)=\sum_{B}(1)$, when $A-C=B-D \geqq 0$ and

$$
R 1 \sum(c+d-a-b)>0 .
$$

In particular, the cases $A=1, B=2, C=D=0$, and $A=B=C=1$, $D=0$, lead to

$$
\begin{aligned}
{ }_{1} F_{1}[a ; b ; z] \sim & \Gamma[1+a-b ; 1-b] z^{-a}{ }_{2} F_{0}[a, 1+a-b ; \quad ;-1 / z] \\
& +e^{z} z^{a-b} \Gamma[b ; a]_{2} F_{0}[1-a, b-a ; \quad ; 1 / z]
\end{aligned}
$$

provided that $|\arg z|<\pi / 2$. This is Barnes' well-known result for the confluent hypergeometric function (see [1]).

2. The analogue for basic functions. I shall now state the corresponding results for basic hypergeometric functions together with an outline of the proof. In the usual notation for basic series, let

$$
{ }_{A} \Phi_{B}[(a) ;(b) ; z]=\sum_{n=0}^{\infty} \frac{\left(a_{1}\right)_{n}\left(a_{2}\right)_{n} \cdots\left(a_{A}\right)_{n} z^{n}}{\left(b_{1}\right)_{n}\left(b_{2}\right)_{n} \cdots\left(b_{B}\right)_{n}(1)_{n}}
$$

where $(a)_{n}=\left(1-q^{a}\right)\left(1-q^{a+1}\right) \cdots\left(1-q^{a+n-1}\right)$, and $|q|<1$. Also let

$$
\prod^{P}[(a) ;(b)]=\prod_{n=0}^{P} \frac{\left(1-q^{a_{1}+n}\right)\left(1-q^{a_{2}+n}\right) \cdots\left(1-q^{a_{A}+n}\right)}{\left(1-q^{b_{1}+n}\right)\left(1-q^{b_{2}+n}\right) \cdots\left(1-q^{b_{B}+n}\right)} .
$$

Let $I_{P, R}$ be the integral

$$
\begin{array}{r}
\frac{1}{2 \pi i} \int \prod^{P}[(a)+s,(b)-s, 1-z+s, z-s ;(c)+s,(d)-s] d s \\
=\int \prod^{P}(s) d s
\end{array}
$$

taken round the contour $A(-i \pi / t) B(i \pi / t) C(R+i \pi / t) D(R-i \pi / t)$, and let $I_{P, R^{\prime}}$ be the same integral taken round the contour $A(-i \pi / t)$ - $B(i \pi / t) E\left(-R^{\prime}+i \pi / t\right) F\left(-R^{\prime}-i \pi / t\right)$. We shall assume now that $P>R$ and $P>R^{\prime}$, and that both contours are indented so that (supposing that $R$ and $R^{\prime}$ are integers) the first $R$ of every ascending sequence of poles of $\Pi^{P}(s)$ fall inside $A B C D$, and the first $R^{\prime}$ of every descending sequence of poles of $\Pi^{P}(s)$ fall inside $A B E F$. We assume also that $q=e^{-t}, t>0$, though the restriction that $q$ is real can easily be removed from the final result by analytic continuation over the circle $|q|<1$.

By the periodicity of the integrand, we have

$$
\int_{B C} \prod^{P}(s) d s=\int_{A D} \prod^{P}(s) d s \text { and } \int_{F \boldsymbol{A}} \prod^{P}(s) d s=\int_{E B} \prod^{P}(s) d s \text {. }
$$


Hence

$$
I_{P, R}=\int_{A B} \prod^{P}(s) d s+\int_{C D} \prod^{P}(s) d s
$$

and

$$
-I_{P, R^{\prime}}=\int_{A B} \prod^{P}(s) d s+\int_{B F} \prod^{P}(s) d s
$$

But

$$
\begin{aligned}
I_{P, R}= & \sum\left(\text { residues within } A B C D \text { of } \prod^{P}(s)\right), \\
= & \frac{1}{t} \sum_{\mu=1}^{D} \prod^{P}\left[\begin{array}{c}
(a)+d_{\mu},(b)-d_{\mu}, 1-z+d_{\mu}, z-d_{\mu} \\
(c)+d_{\mu},(d)^{\prime}-d_{\mu}, 1
\end{array}\right] \\
& \times \sum_{n=0}^{R} \frac{\left((c)+d_{\mu}\right)_{n}\left(1+d_{\mu}-(b)\right)_{n} Q^{n}}{\left((a)+d_{\mu}\right)_{n}\left(1+d_{\mu}-(d)\right)_{n}}
\end{aligned}
$$

where $Q=\left(-q^{\left.(n+1) / 2+d_{\mu}\right)(D-B)} q^{b_{1}+\cdots+b_{B}-d_{1}-\cdots-d_{D}+z}\right.$, that is

$$
I_{P, R}=\sum^{D} \prod^{P} \sum^{R}(d), \text { say. }
$$

Similarly,

$$
\begin{aligned}
-I_{P, R^{\prime}}= & \frac{1}{t} \sum_{\nu=1}^{c} \prod_{\nu=1}^{P}\left[\begin{array}{c}
(b)+c_{\nu},(a)-c_{\nu}, z+c_{\nu}, 1-z-c_{\nu} \\
(d)+c_{\nu},(c)^{\prime}-c_{\nu}, 1
\end{array}\right] \\
& \times \sum_{n=0}^{R^{\prime}} \frac{\left((d)+c_{\nu}\right)_{n}\left(1+c_{\nu}-(a)\right)_{n} Q^{\prime n}}{\left((b)+c_{\nu}\right)_{n}\left(1+c_{\nu}-(c)\right)_{n}}
\end{aligned}
$$

where $Q^{\prime}=\left(-q^{(n+1) / 2+c \nu}\right)^{(C-A)} q^{a_{1}+\cdots+a_{A}-c_{1}-\cdots-c_{C}+1-z}$, that is,

$$
-I_{P, R^{\prime}}=\sum^{C} \prod^{P} \sum^{R^{\prime}}(c), \text { say, }
$$

and so

$$
\begin{aligned}
\int_{A B} \prod^{P}(s) d s & =\sum^{D} \prod^{P} \sum^{R}(d)+\int_{D C} \prod^{P}(s) d s \\
& =\sum^{c} \prod^{P} \sum^{R^{\prime}}(c)+\int_{F E} \prod^{P}(s) d s
\end{aligned}
$$

Now

$$
\left|\int_{D C} \prod^{P}(s) d s\right| \leqq \frac{1}{2 \pi} \int_{-\pi / t}^{\pi / t}\left|\prod^{P}(R+i r)\right| d r
$$


and

$$
\left|\int_{F E} \prod^{P}(s) d s\right| \leqq \frac{1}{2 \pi} \int_{-\pi / t}^{\pi / t}\left|\prod^{P}\left(-R^{\prime}+i r\right)\right| d r .
$$

It has been shown previously (Slater [3]) that if $D=B$ and $\Re \sum(b-d)>0$, or if $D>B, \int_{A B} \prod^{P}(s) d s=\sum^{D} \prod^{\infty} \sum^{\infty}(d)$. Also if $A=C$ and $\Re \sum(a-c)>0$, or if $C>A$,

$$
\int_{A B} \prod^{P}(s) d s=\sum^{c} \prod^{\infty} \sum^{\infty}(c) \text {. }
$$

In all cases, even when $C<A$, or when $D<B$, we have for $R$ fixed

$$
\begin{aligned}
& \left|\int_{D C} \prod^{P}(s) d s\right| \\
& \quad \leqq \frac{1}{t} \prod^{\infty} \frac{\left|1+q^{(a)+R+n}\right|\left|1+q^{1-z+R+n}\right|\left|1+q^{(b)-R+n}\right|\left|1+q^{z-R+n}\right|}{\left|1-q^{(c)+R+n}\right|\left|1-q^{(d)-R+n}\right|} .
\end{aligned}
$$

But the next term of the series $\sum^{D} \prod^{P} \sum^{R}(d)$ would be

$$
\begin{array}{r}
\sum_{\mu=1}^{D} \prod^{P}\left[\begin{array}{c}
(a)+d_{\mu}, 1-z+d_{\mu},(b)-d_{\mu}, z-d_{\mu} \\
(c)+d_{\mu},(d)^{\prime}-d_{\mu}, 1
\end{array}\right] \\
\times \frac{\left((c)+d_{\mu}\right)_{R+1}\left(1+d_{\mu}-(b)\right)_{R+1} Q^{R+1}}{\left((a)+d_{\mu}\right)_{R+1}\left(1+d_{\mu}-(d)\right)_{R+1}}
\end{array}
$$

which is of the same order in $R$ as $\int_{D C} \prod^{P}(s) d s$. Similarly, for $R^{\prime}$ fixed $\int_{F E} \prod^{P}(s) d s$ is also bounded above as $P \rightarrow \infty$, and this integral is of the same order in $R^{\prime}$ as the $\left(R^{\prime}+1\right)$ th term of the series $\sum c \prod^{P} \sum^{R^{\prime}}(c)$.

Hence we have

$$
\begin{aligned}
\frac{1}{2 \pi i} \int_{-i \pi / t}^{i \pi / t} \prod^{\infty}[(a)+s, 1-z+s,(b)-s, z-s ;(c)+s,(d)-s] d s \\
\sim \frac{1}{t} \sum_{\mu=1}^{D} \prod^{\infty}\left[\begin{array}{c}
(a)+d_{\mu}, 1-z+d_{\mu},(b)-d_{\mu}, z-d_{\mu} \\
(c)+d_{\mu},(d)^{\prime}-d_{\mu}, 1
\end{array}\right] \\
\quad \times_{B+C} \Phi_{A+D-1}\left[\begin{array}{c}
(c)+d_{\mu}, 1+d_{\mu}-(b) ; \\
(a)+d_{\mu}, 1+d_{\mu}-(d)^{\prime} ;
\end{array}\right] \\
\sim \frac{1}{t} \sum_{\nu=1}^{c} \prod^{\infty}\left[\begin{array}{c}
(b)+c_{\nu},(a)-c_{\nu}, z+c_{\nu}, 1-z-c_{\nu} \\
(d)+c_{\nu},(c)^{\prime}-c_{\nu}, 1
\end{array}\right] \\
\quad \times_{A+D} \Phi_{B+C-1}\left[\begin{array}{c}
(d)+c_{\nu}, 1+c_{\nu}-(a) ; \\
(b)+c_{\nu}, 1+c_{\nu}-(c)^{\prime} ;
\end{array}\right]
\end{aligned}
$$


where

$$
Q=\left(-q^{\left.(n+1) / 2+d_{\mu}\right)(D-B)} q^{b_{1}+\cdots+b_{B}-d_{1}-\cdots-d_{D}+z},\right.
$$

and

$$
Q^{\prime}=\left(-q^{\left.(n+1) / 2+c_{\nu}\right){ }^{(C-A)}} q^{a_{1}+\cdots+a_{A}-c_{1}-\cdots-c_{C}+1-z},\right.
$$

even when $D<B$ or when $C<A$.

In particular, let

$$
A=B=0, \quad C=1, \quad D=2,
$$

then

$$
\begin{aligned}
\frac{1}{2 \pi i} \int_{-i \pi / t}^{i \pi / t} & \prod^{\infty}[1-x+s, x-s ; a+s, 1-b-s,-s] d s \\
= & \prod^{\infty}\left[\begin{array}{l}
1-x, x \\
a, 1-b, 1
\end{array}\right]{ }_{1} \Phi_{1}\left[a ; b ;-q^{(n+1) / 2+x+b-1}\right] \\
& +\prod^{\infty}\left[\begin{array}{l}
2-b-x, x-1,+b \\
1+a-b, b-1,1
\end{array}\right] \\
& \times_{1} \Phi_{1}\left[1+a-b ; 2-b ;-q^{(n+1) / 2+x}\right\rfloor \\
\sim & \prod^{\infty}\left[\begin{array}{l}
x+a, 1-x-a \\
1+a-b, a, 1
\end{array}\right]{ }_{2} \Phi_{0}\left[1+a-b, a ; ; q^{1-x-a}\right]
\end{aligned}
$$

and, if $A=1=C=D, B=0$, then

$$
\begin{aligned}
& \frac{1}{2 \pi i} \int_{-i \pi / t}^{i \pi / t} \prod^{\infty}[b+s, 1-x+s, x-s ; a+s,-s] d s \\
&=\prod^{\infty}\left[\begin{array}{l}
b, 1-x, x \\
a, 1
\end{array}\right]{ }_{1} \Phi_{1}\left[a ; b ; q^{x}\right] \\
& \sim \prod^{\infty}\left[\begin{array}{l}
b-a, 1-x-a, x+a \\
a, 1
\end{array}\right] \\
& \times{ }_{2} \Phi_{0}\left[a, 1+a-b ; ;-q^{1+b-2 a-x-(n+1) / 2}\right]
\end{aligned}
$$

But

$$
\text { (2.5) } \begin{aligned}
& { }_{1} \Phi_{1}\left[a ; b ; q^{(n+1) / 2+x}\right] \\
& =\prod^{\infty}[1+a-b+x+\pi i / t]_{1} \Phi_{1}\left[b-a ; b ;-q^{1+a+x-b}\right]
\end{aligned}
$$

and

(2.6) ${ }_{1} \Phi_{1}\left[a ; b ; q^{x}\right]=1 / \prod^{\infty}[x] \cdot{ }_{1} \Phi_{1}\left[b-a ; b ;-q^{(n+1) / 2+x+a-1}\right]$. 
Hence we have

$$
\begin{aligned}
{ }_{1} \Phi_{1}[a ; b ; & \left.q^{(n+1) / 2+x}\right] \\
\sim & \prod^{\infty}\left[\begin{array}{l}
1+a-b+x+\pi i / t, b-a-x+\pi i / t, 1-b \\
1+a-b, b+\pi i / t-x, 1-b+x+\pi i / t
\end{array}\right] \\
& \times{ }_{2} \Phi_{0}\left[a, 1+a-b ; ;-q^{b-a-x}\right] \\
& +\prod_{\prod}^{\infty}\left[\begin{array}{l}
x+1+\pi i / t, \pi i / t-x, a \\
b, 1+a-b+x+\pi i / t, b-a-x+\pi i / t
\end{array}\right] \\
& \times{ }_{2} \Phi_{0}\left[\begin{array}{l}
\left.1-a, b-a ; ; q^{a-x-(n+1) / 2}\right]
\end{array}\right.
\end{aligned}
$$

and

$$
\begin{aligned}
\sim & \prod^{\infty}\left[\begin{array}{l}
2 a-1-x, 1-b \\
1-a, 1+b-a-x, a-b+x
\end{array}\right] \\
& \times{ }_{2} \Phi_{0}\left[\begin{array}{l}
\left.1-a, b-a ; ; q^{1-x}\right] \\
+
\end{array}\right. \\
+ & \prod^{\infty}\left[\begin{array}{l}
a+x, 1-a-x, b-a \\
x, b, x, 1-x
\end{array}\right] \\
& \times{ }_{2} \Phi_{0}\left[1+a-b, a ; ;-q^{1-2 a+b-x-(n+1) / 2}\right] .
\end{aligned}
$$

These two results are the analogues for basic series of (1.4) above.

\section{REFERENCES}

1. E. W. Barnes, The asymptotic expansions of integral functions defined by generalised hypergeometric series, Proc. London Math. Soc. (2) vol. 5 (1907) pp. 59-116.

2. C. S. Meijer, On the G-function, I-VIII, Proc. Amsterdam Akad. vol. 49 (1946).

3. L. J. Slater, An integral of hypergeometric type, Proc. Cambridge Philos. Soc. vol. 48 (1952) pp. 578-582.

4. G. N. Watson, The continuation of functions defined by generalised hypergeometric series, Trans. Cambridge Philos. Soc. vol. 21 (1910) pp. 281-299.

5. E. M. Wright, The asymptotic expansion of the generalised hypergeometric functions, J. London Math. Soc. vol. 10 (1935) pp. 286-293.

Newnham College, Cambridge University 